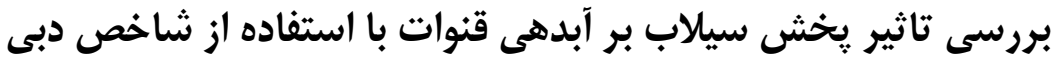
استاندارد شده (مطالعه موردى : قنوات منطقه ميانكوه مهريز)

محمدرضا فاضل :ور عقدائى'، حسين ملكى نزاد'، محمدرضا اختصاصى 'و جلال برخوردارى ع

1- دكترى آبخيزدارى، اداره كل منابع طبيعى و آبخيزدارى يزد، (نويسنده مسوول: fazelpoor_reza@yahoo.com

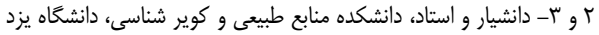

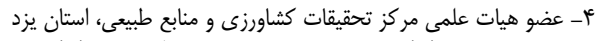

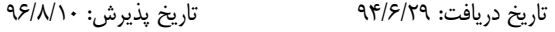

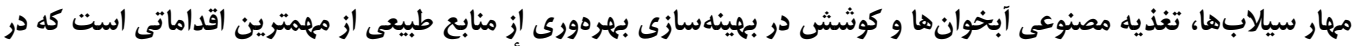

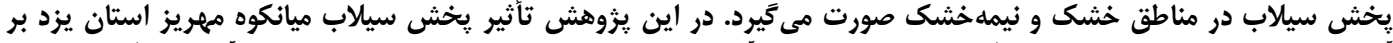

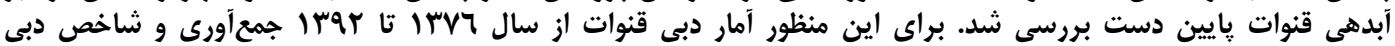

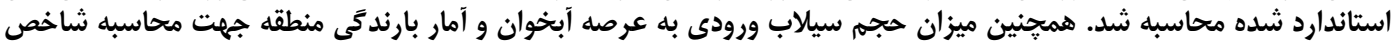

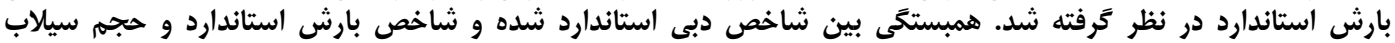

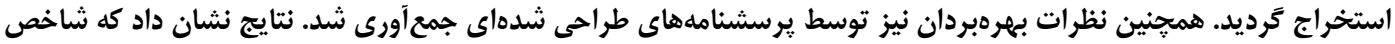

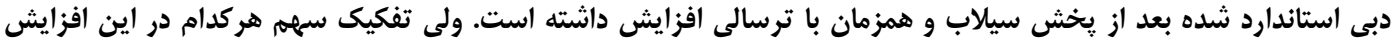

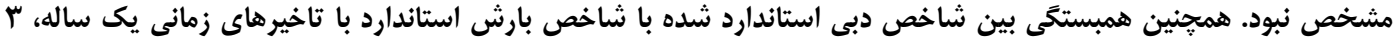

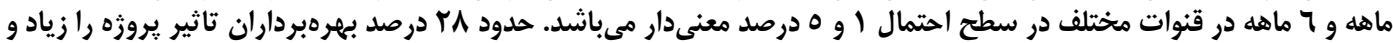

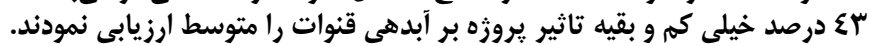

وازههاى كليدى: آبخوان، تغذيه، دبى، سيلاب، يزد

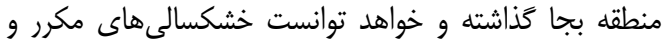

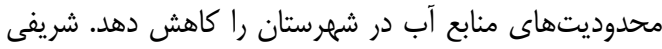

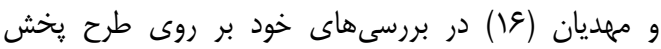

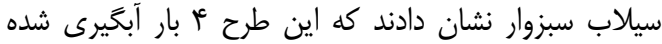

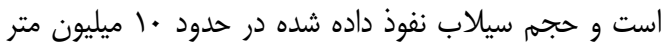

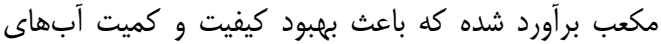

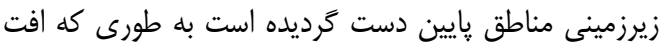

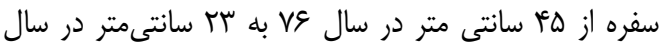
كاهش يافته است. خلجى (9) اثرات طرحهاى يخش استش سيلاب بر آبهاى

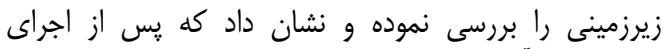

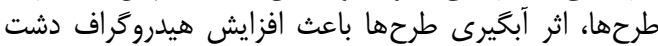

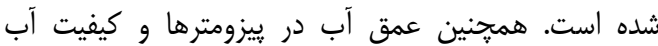

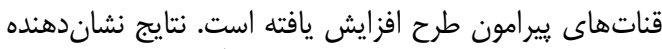

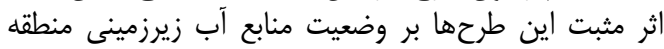

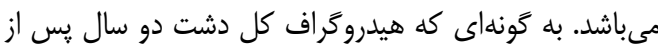

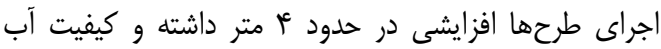

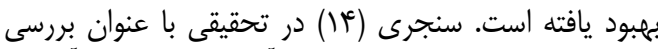

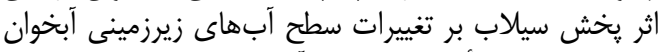

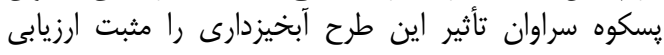

$$
\text { نمودند. }
$$

بيات موحد و شامى (1) تأثير يخش سيلاب دشت سمرين

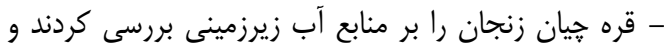

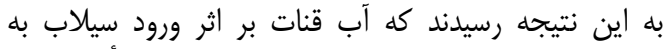

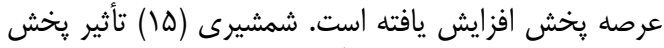

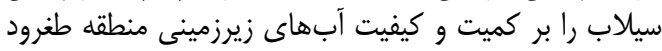

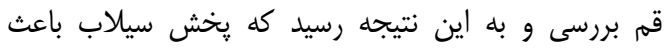

مقدمه

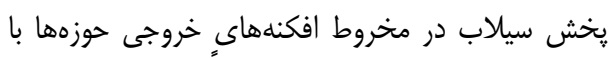

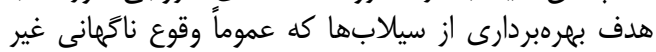

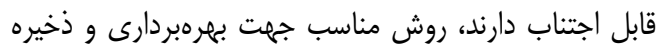

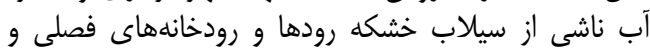

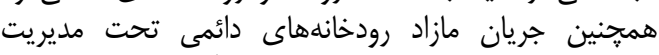

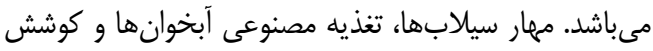

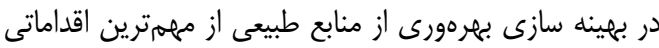

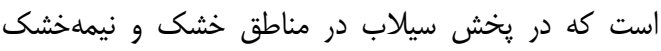

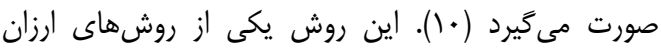

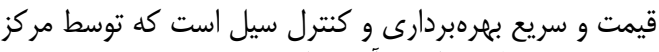
تحقيقات حفاظت خاك و آبخيزدارى توسعه يافتئه

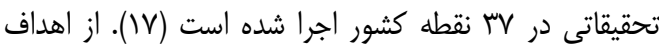

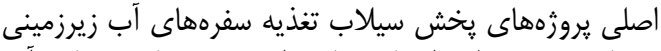

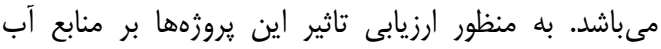

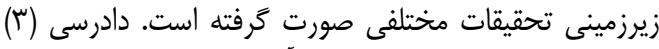

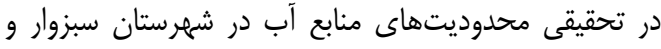

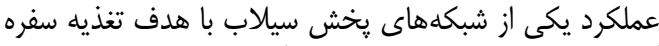

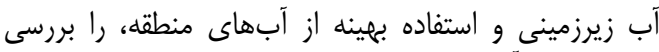

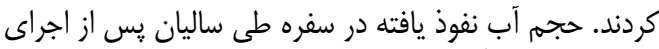

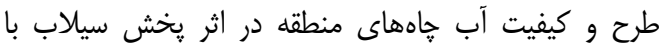

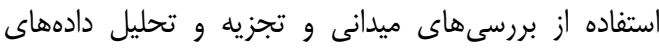

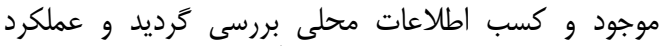

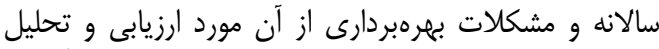

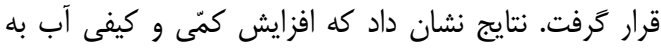

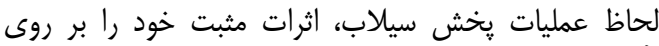

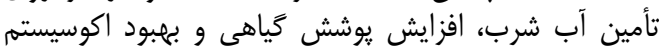


رسيدند كه اين طرح شرايط كيفى و كمى آبخوان را بهبود بخشيده است. هاشمى و همكاران (V) در مطالعهاى به بررسى نقش تغذيه

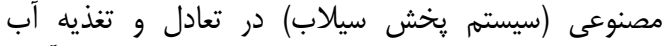

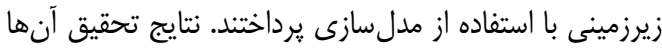

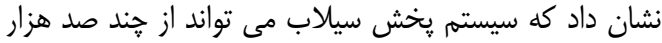

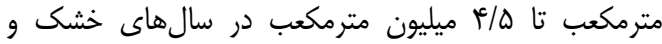

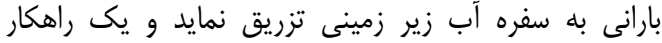

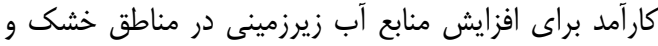

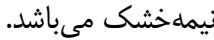

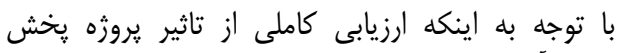

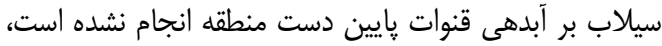

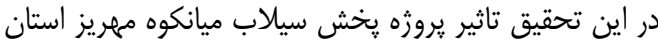

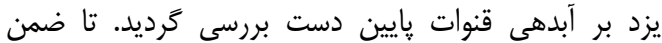

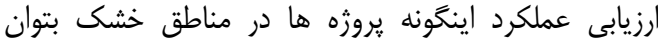

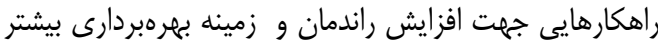

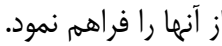

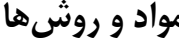
منطقه مورد مطالعه

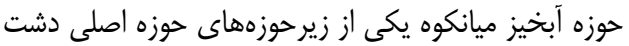

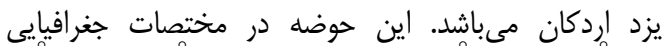

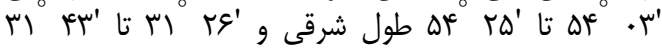

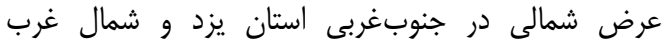

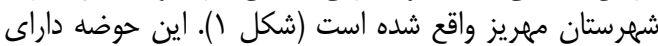

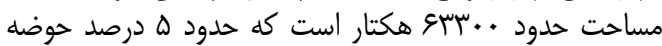

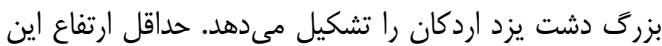

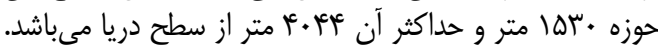

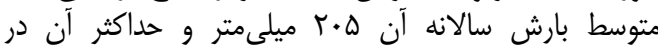

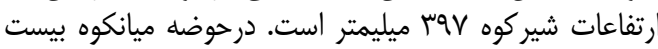

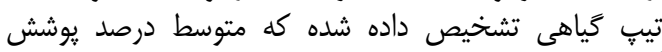

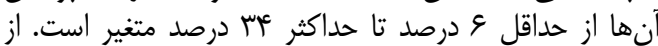

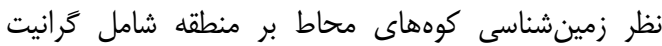

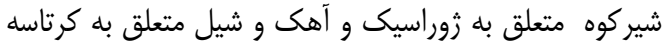

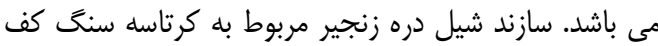

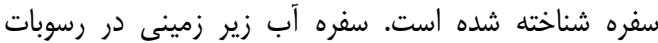
آبرفتى كه ضخامت آن گأهى به به بيش از صد متر مىرسد، قرار

معرفى سيستم يخش سيلاب ميانكوه مهريز

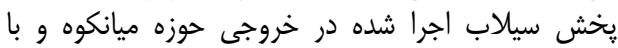

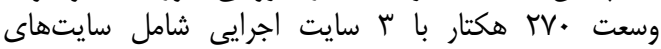

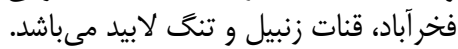

افزايش دبى قنوات و سطح آب خاهها و افزايش محصولات كشاورزى و كاهش مهراجرت شده است است

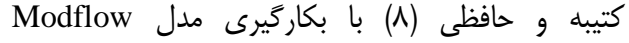

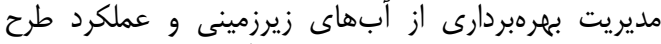

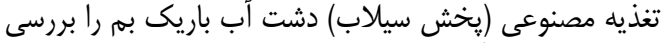

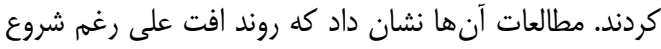

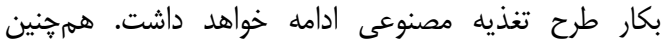

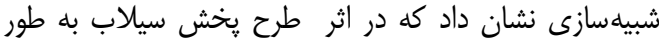

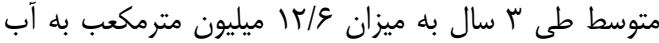

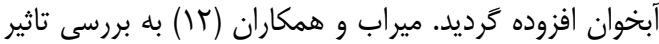

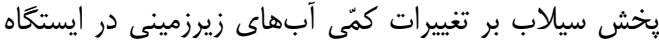

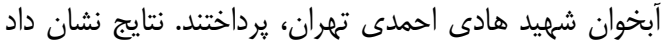

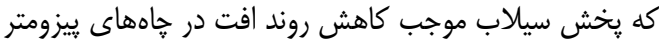

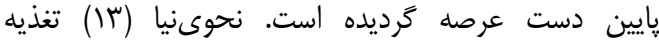

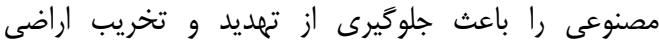

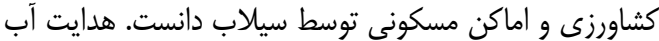

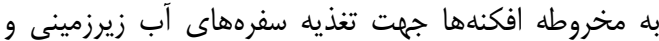

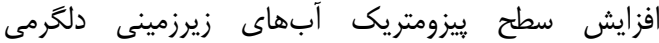

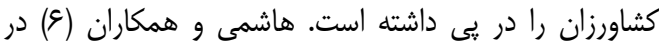

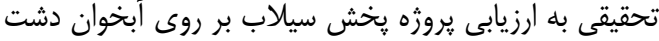

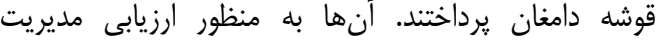

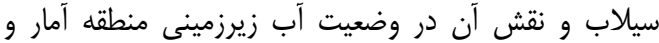

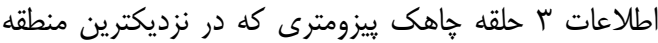

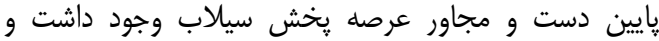
همجنين سه حلقه جاه يِيزومترى ديكر استفاده نمودند. نتايج

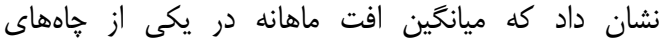

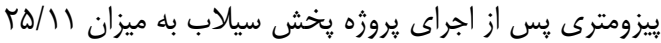
درصد كاسته شده است.

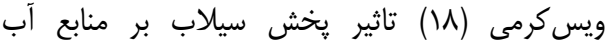

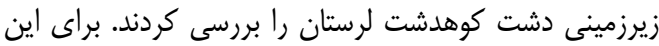

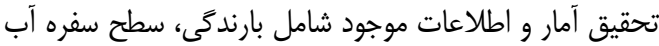

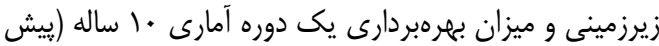

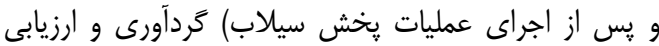

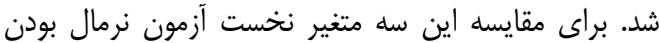

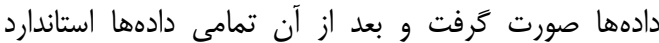

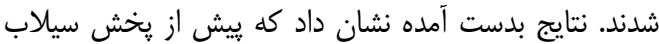
(تان سال VQ-VG)

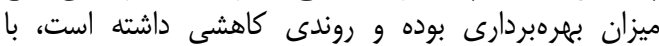

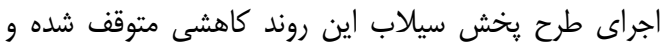
سطح آب زيرزمينى افزايش داشته سئ است.

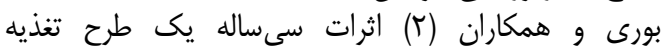
مصنوعى را در تبوليا مورد بررسى قرار دات دادند و به اين نتيجه 


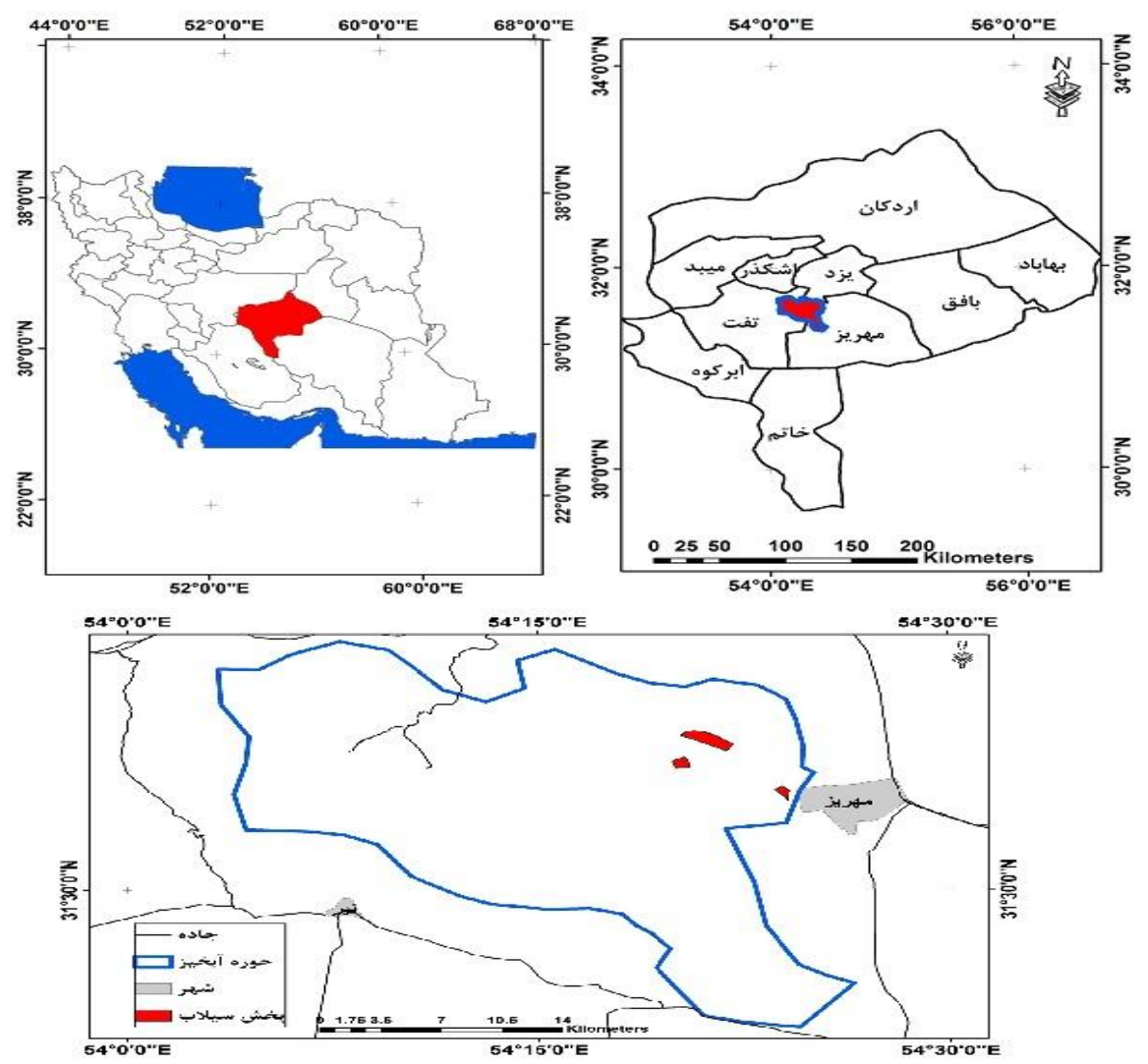

شكل 1- موقعيت منطقه مورد مطالعه

Figure 1. Location of the study area

$$
\text { SPI }=\frac{P_{i}-\bar{P}}{\sigma} \quad \text { (T) }
$$
كه در آن معيار دادهها مىباشد.

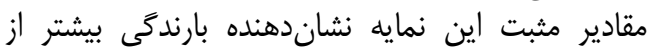

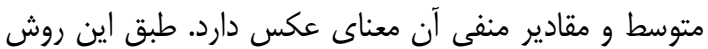

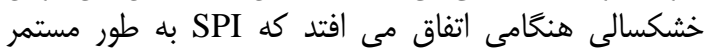

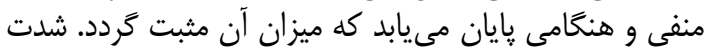

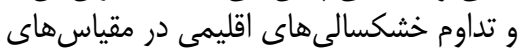

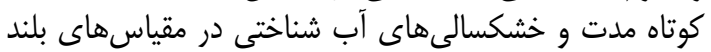

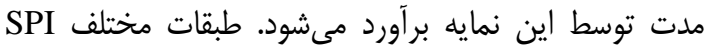

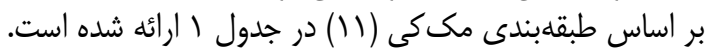

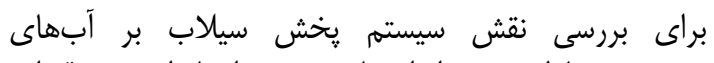
زيرزمينى مناطق همجوار از شاخص دبى استاندارد شده قناب إنوات

$$
S D I=\frac{Q_{i}-\bar{Q}}{\sigma}
$$

كه در اين فرمول و و مانحر اف معيار دادهها مى فياشد.

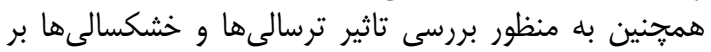

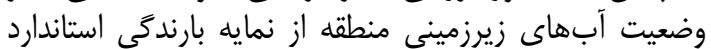

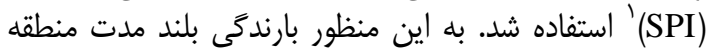

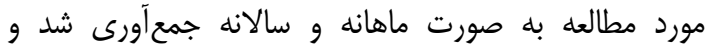
شاخص بارش استاندارد از رابطه ب محاسبه كَرديد.

SPI جدول (- طبقهبندى شاخص

Table 1. Classification of the SPI Index

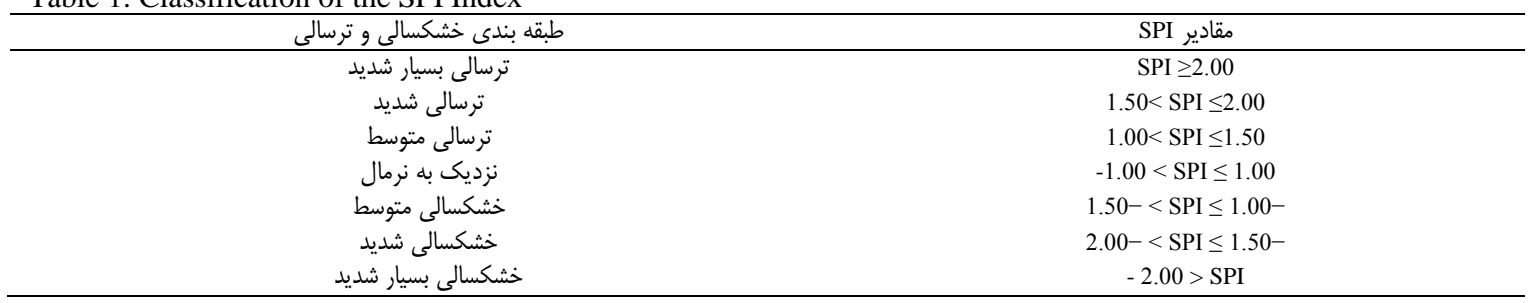


r نشان داده شد. به منظور بررسى تاثير خشكسالى و ترسالى

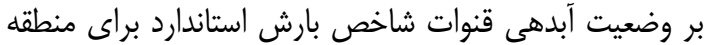

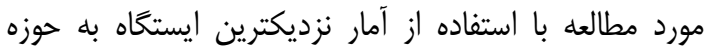

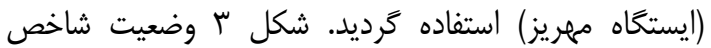

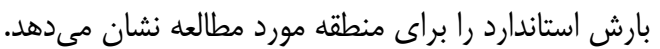

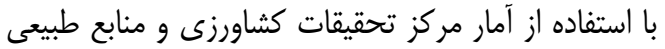

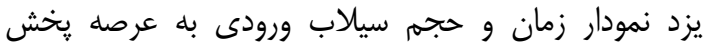

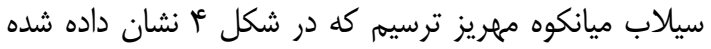

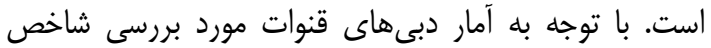

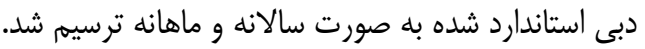

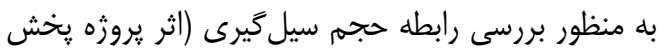

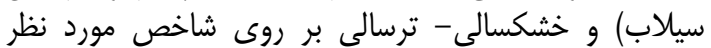

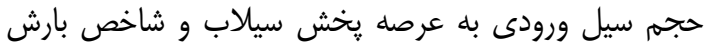

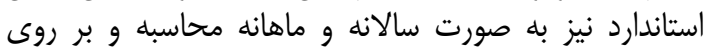

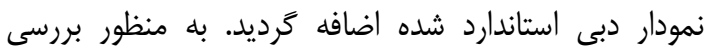

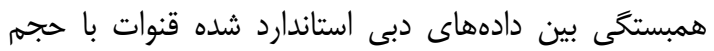

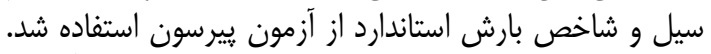

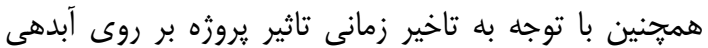

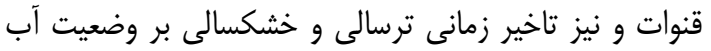

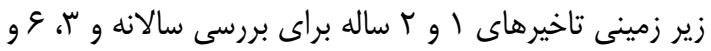

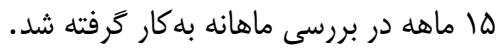

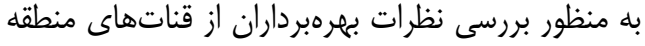

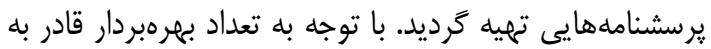

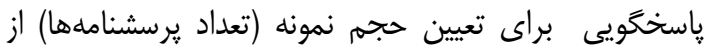

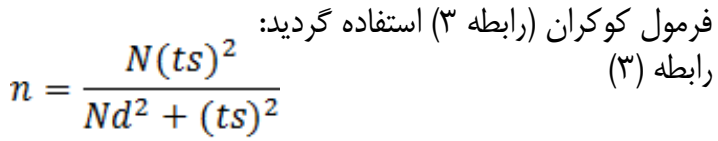
N جامعه آمارى، n: : نمونه آمارى، t: سطح اطمينان

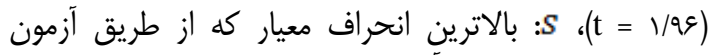

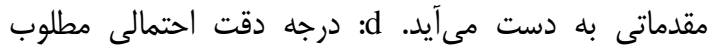

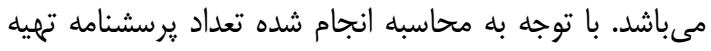

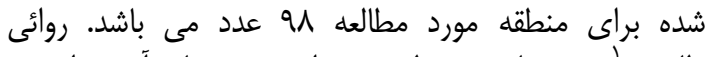

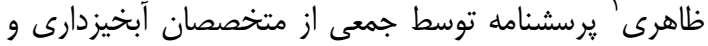

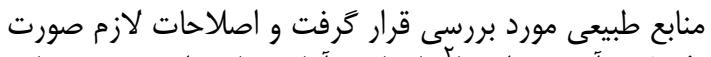

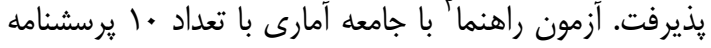

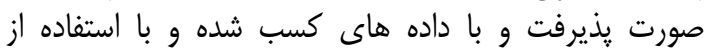

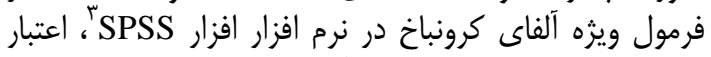

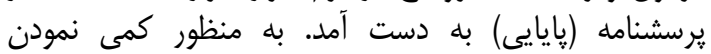

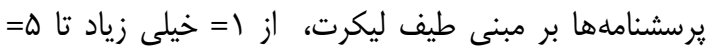
خيلى كم طراحى كَرديد.

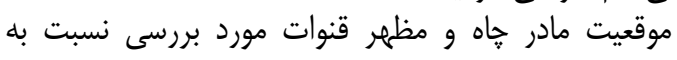
محل اجراى يروزه يخش سيلاب در منطقه به صورت شكل 

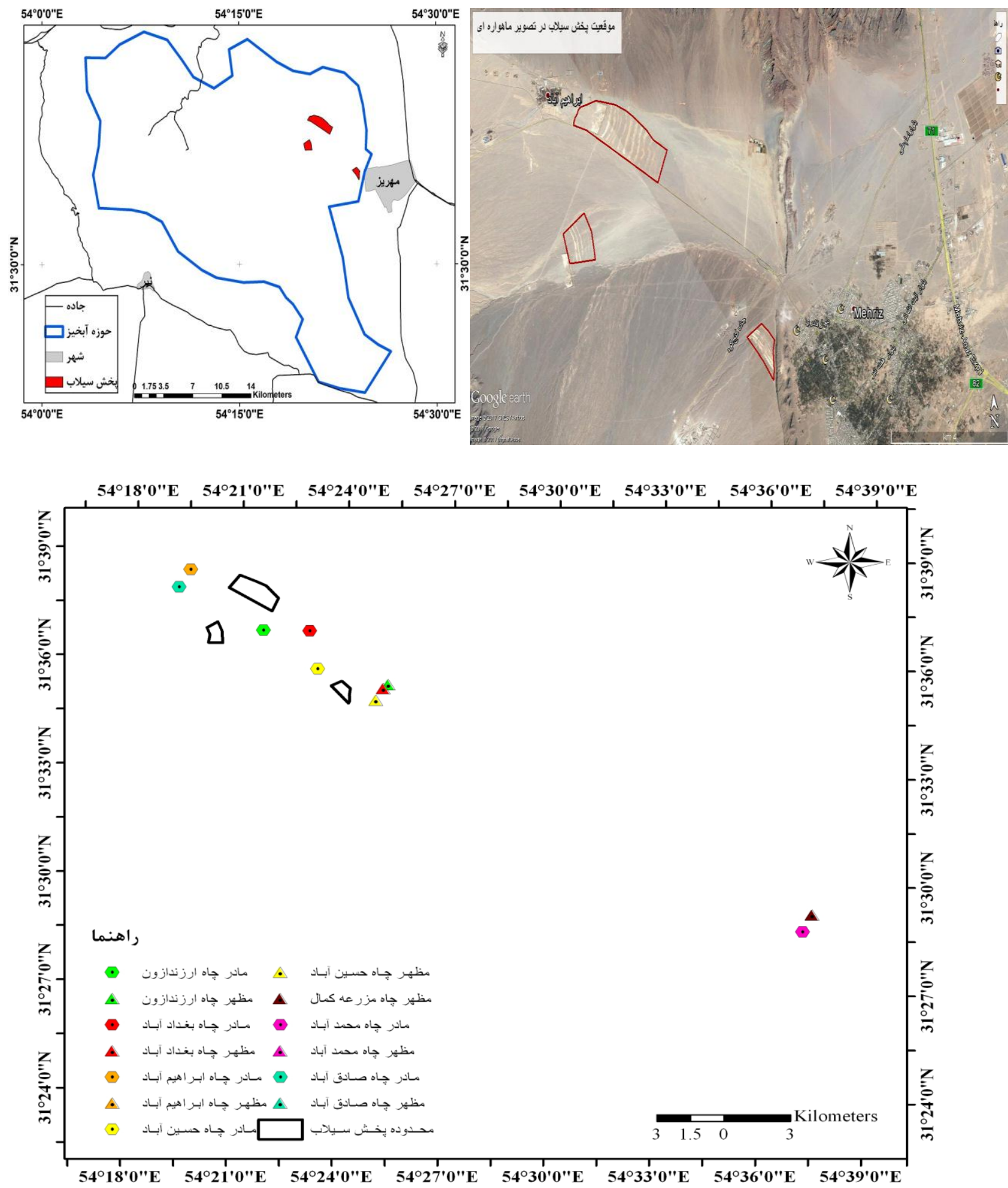

شكل r- موقعيت قنوات مورد بررسى نسبت به محدوده يخش سيلاب

Figure 2. The position of the Qanats in relation to the flood spreading area 


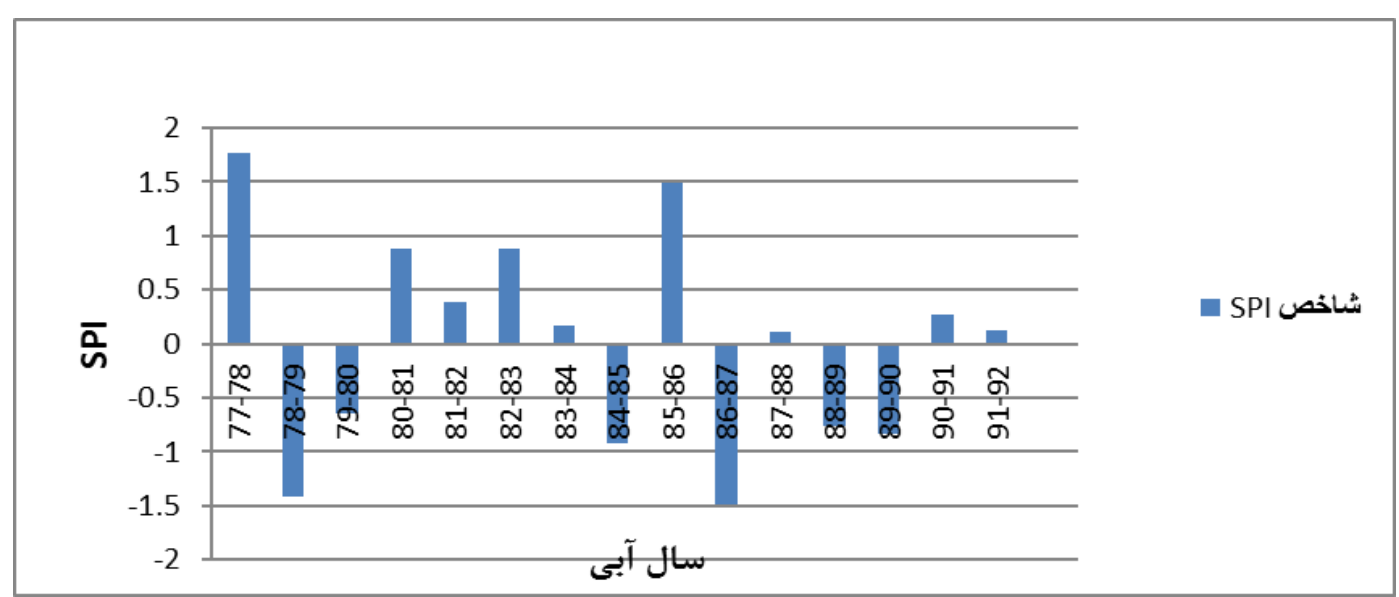

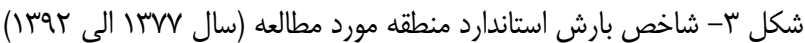

Figure 3. Standard rainfall index of the study area (1998 to 2013)

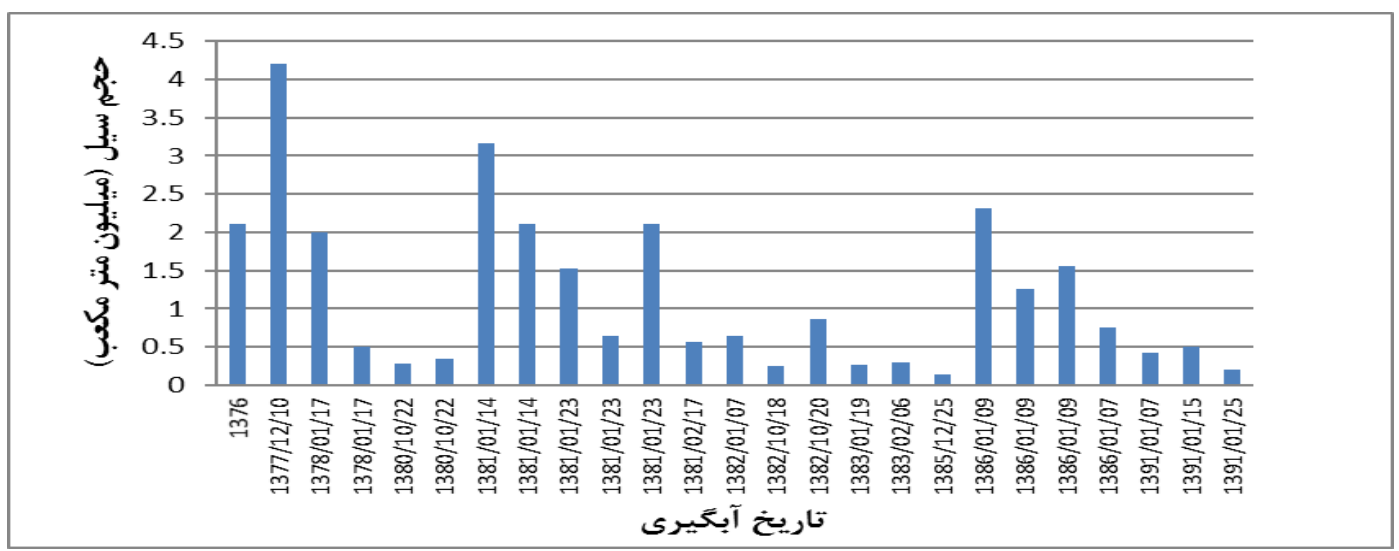

شكل rأ- تاريخ آبخيرى و حجم سيلاب عرصه يخش سيلاب ميانكوه مهريز

Figure 4. Flooding date and flood volume in the Myankooh Mehriz flood spreading area

دادههاى ماهانه زياد بوده و نمايش آن به صورت نمودار

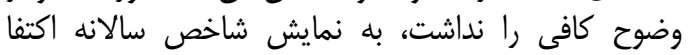
كرديد. جداول ع ا تا V همبستخى محاسبه شده را نشان مى دهد.

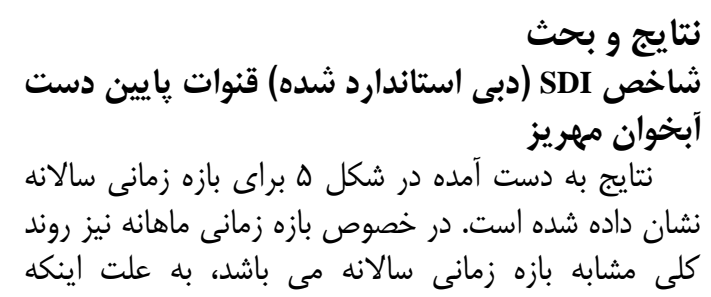




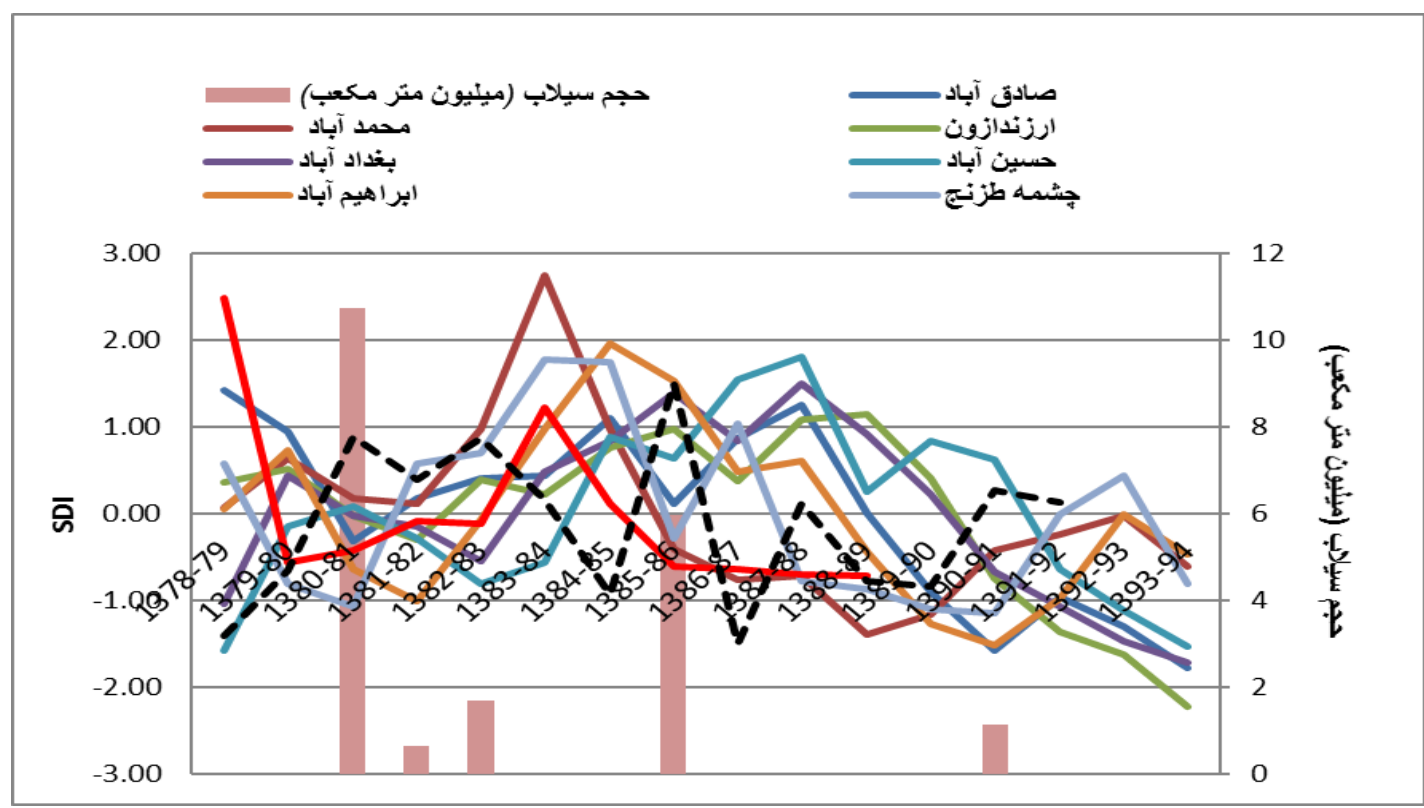

شكل ه- نمودار SDI سالانه قنوات مجاور يخش سيلاب ميانكوه مهريز

Figure 5. Annual SDI diagram of the Qanats around the Myankooh Mehriz flood spreading

است. همجنين جدول پايايى برسشنامهها را بر اساس ميزان

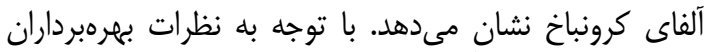

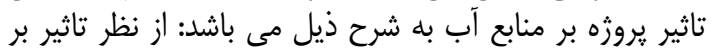

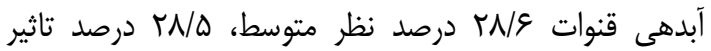

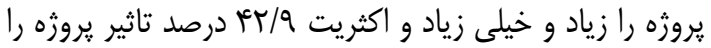

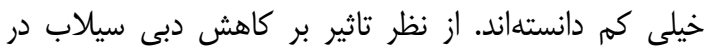

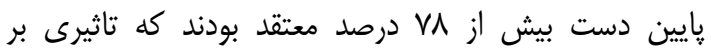

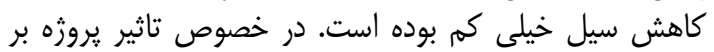

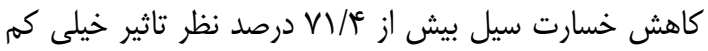

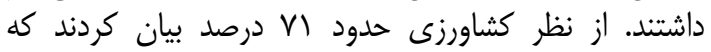

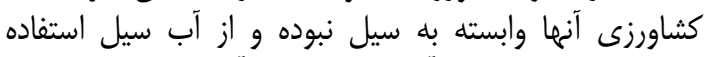

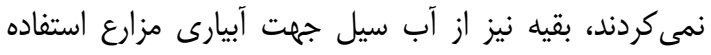

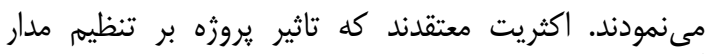
آبيارى، كاهش سطح اراضى كشاورزى و الخوى كشت متر متوسط

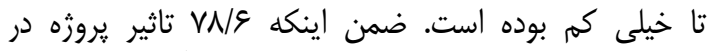

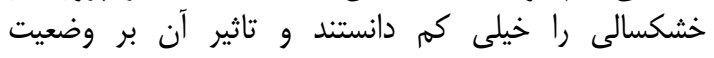
اقتصادى و اجتماعى بهرهبرداران متوسط تانيل خيلى خيلى كم اعلام

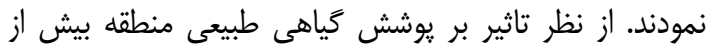

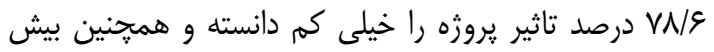

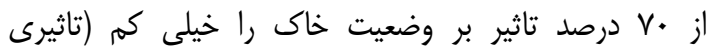

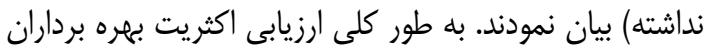

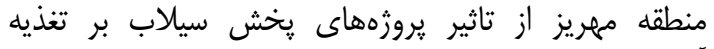
أبخوان خيلى كم، نتيجه كَيرى شد.

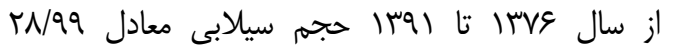

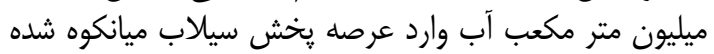

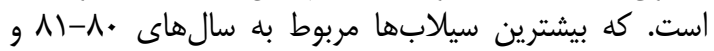

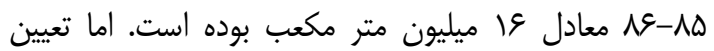

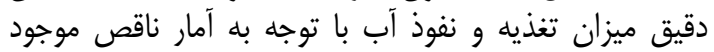

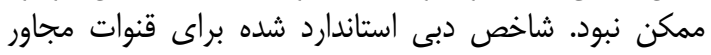

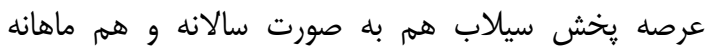

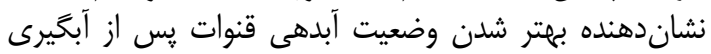

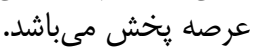

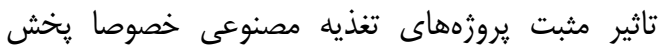

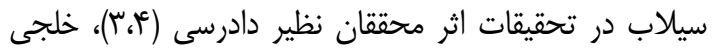

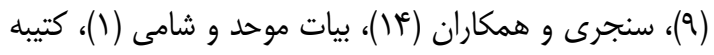

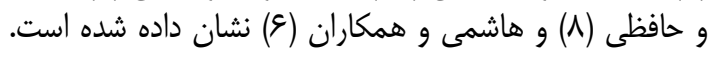

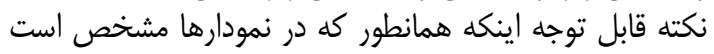

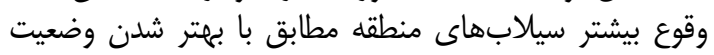

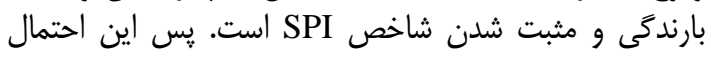

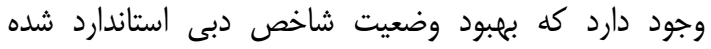

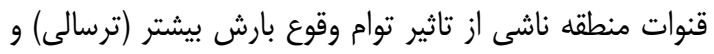

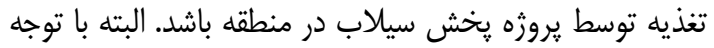

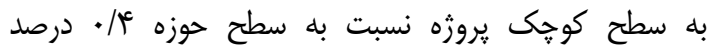
تفكيك اين تاثير بر تغذيه سفره كار بسيار دشوارى بهر است. نتايج حاصل از يرسشنامهنهائ بهره بره بردار بردار بران مشخصات بجره برداران منطقه در جدول بران ارائه شده بهان 
Table 2. Characteristics of the operators of the Mehriz area

\begin{tabular}{|c|c|c|c|}
\hline درصد تجمعى & درصد & كروه & صفت \\
\hline$T I / T^{c}$ & $r I / 4$ & $r \cdot-r$. & \multirow{4}{*}{ سن (سال) } \\
\hline FT/A & $r M / s$ & $f \cdot-\Delta$. & \\
\hline $1 . \cdot$ & $\Delta V / T$ & $>\Delta$. & \\
\hline & $1 \cdots$ & مجموع & \\
\hline$r \Delta / V$ & $r \Delta / V$ & $<r$. & \multirow{3}{*}{ سابقه كشاورزى } \\
\hline $94 / \%$ & TN/S & $r \cdot-\varphi$. & \\
\hline $1 . \cdot$ & $r \Delta / v$ & $>r$. & \\
\hline$V / / F$ & $V \backslash / f$ & $<r$. & \multirow{4}{*}{ سابقه دامدارى } \\
\hline $9 T / 1$ & $r M / F$ & $r \cdot-\boldsymbol{r}$. & \\
\hline $1 .$. & $V / r$ & $>r$. & \\
\hline & $1 \cdots$ & مجموع & \\
\hline$V N / 9$ & $V N / S$ & بى سواد، ابتدايى & \multirow{4}{*}{ ميزان تحصيلات } \\
\hline$\Lambda \Delta / V$ & $V / I$ & راهنمايى و متوسطه & \\
\hline $9 \% / 9$ & $\mathrm{~V} / \mathrm{I}$ & ليسانس & \\
\hline $1 .$. & $\mathrm{V} / \mathrm{I}$ & فوق ليسانس & \\
\hline
\end{tabular}

جدول r- مشخصات بهره برداران منطقه مهريز

Table 3. Cronbach's alpha coefficient (reliability) Mehriz questionnaire

جدول ب- ضريب آلفاى كرونباخ (يايايى) يرسشنامهاى مهريز ألفا كرونباخ أفا كرونباخ براى دادههاى استاندارد شده تعداد دادهها $\cdot / \wedge \mathrm{r}^{2}$

$\cdot / \Lambda \mathrm{IV}$

جدول ع- همبستخى بين شاخص SDI سالانه قنوات اطراف يخش سيلاب مهريز با شاخص SPI و حجم سيلاب ورودى به آبخوان Table 4. Correlation between Annual SDI Index of Qanats around the Mehriz Flood spreading with the SPI Index and Flood Volume in the Aquifer

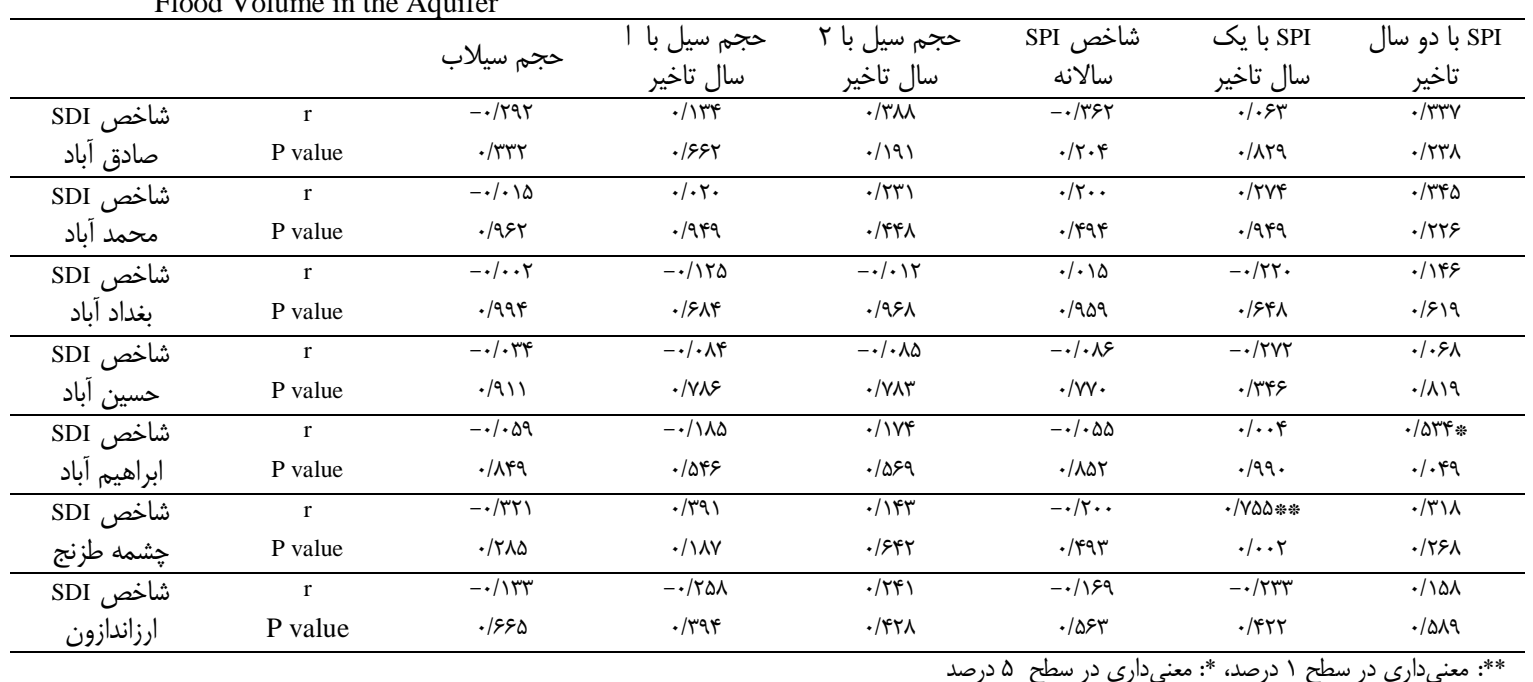


جدول ه- همبستخى بين شاخص SDI سالانه قنوات اطراف يخش سيلاب مهريز با يكديخر Table 5. Correlation between Annual SDI Index of Qanats around the Mehriz Mehriz Flood spreading

\begin{tabular}{|c|c|c|c|c|c|c|c|c|}
\hline & & شاخص آباد & شاخص SDI آباد & 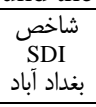 & شاخص SDI & شاخص ابراهيم آباد & $\begin{array}{l}\text { شاخص طشم طزنج } \\
\text { شاخم }\end{array}$ & $\begin{array}{l}\text { شاخص ارزاندازون } \\
\text { SDI }\end{array}$ \\
\hline \multirow{2}{*}{ شاخص آباد } & $\mathrm{r}$ & 1 & $\cdot / \mu \cdot \Lambda$ & $\cdot / 9 \cdot 1$ & $\cdot /$ rqf &. $\mid a+\lambda$ & $\cdot / F \Delta T$ & $\cdot / \mathrm{VW}$ \\
\hline & $P$ value & & . MGA & $\cdot / \cdot 1 r^{*}$ & . / & $\cdot / \cdot v^{*}$ &.$/ \cdot v q$ &.$/ . *^{*}$ \\
\hline \multirow{2}{*}{ شاخص آباد } & $\mathrm{r}$ & $\cdot / \mu \cdot \Lambda$ & 1 & $-\cdot / \cdot r \Delta$ & I- & . & $\cdot / 990 *$ &.$/ . \mu_{q}$ \\
\hline & $P$ value & . MGF & & . /9rg & - ITTA & $.1 \cdot 19$ & $\cdot 1 \cdots \Delta$ & . /MV \\
\hline \multirow{2}{*}{ شغداد آباد } & $\mathrm{r}$ & $\cdot / 9 \cdot 1^{*}$ &.$- / . r \Delta$ & 1 & $\cdot|\wedge|{ }^{*}$ & $\cdot / \Delta F V^{*}$ & $.1 \cdot 49$ & 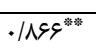 \\
\hline & $P$ value & .1 .14 & . & & . & $\cdot 1 \cdot r \Lambda$ & $\cdot / \Lambda \& D$ & . \\
\hline \multirow{2}{*}{ شاخص آباد } & $\mathrm{r}$ & . Taq & $-\cdot / Y Y I$ & $\cdot|\wedge| \cdot{ }^{*}$ & 1 &.$/$ IIV &.$- / N G Y$ & $\cdot / \Delta Q Y^{*}$ \\
\hline & $P$ value & . & . & . & &.$|F Y|$ & $. / 4 .$. &.$/ .18$ \\
\hline \multirow{2}{*}{ شاخص ابراهيم آباد } & $\mathrm{r}$ & $\cdot / 94 \Lambda^{* * 3}$ & . & $\cdot / \Delta)^{C} V_{*}$ &.$/ T I V$ & 1 & ./DFF" & $\cdot / F A T$ \\
\hline & $P$ value &.$/ . v V$ & $.1 \cdot 19$ &.$/ . r \Lambda$ & | & & r & .1 .09 \\
\hline \multirow{2}{*}{ شاخص طازنج } & $\mathrm{r}$ & $\cdot / F \Delta T$ &.$/ 99 D^{* * *}$ & $.1 .+8$ &.$- / N F T$ & . $/ \Delta F^{\prime \prime \prime}$ & 1 & .1 .90 \\
\hline & $P$ value & $.1 . v 9$ &.$/ \cdot \Delta$ & $\cdot \mid \Lambda E \Delta$ & .19. & . & &.$/ N T r$ \\
\hline \multirow{2}{*}{ شاخص ارزاندازون } & $\mathrm{r}$ & $\cdot / V V^{* * *}$ &.$/ . r q$ & $\cdot / \Lambda \Lambda \gamma^{* * *}$ & $\cdot / \Delta 9 Y^{\prime \prime}$ & . $/$ \&AY & .1 .90 & 1 \\
\hline & $P$ value & . & . IMAV & . & .1 .19 & $.1 . \Delta q$ & ./VTV & \\
\hline
\end{tabular}

جدول 9 - همبستخى بين شاخص SDI ماهانه قنوات اطراف يخش سيلاب مهريز با شاخص SPI و حجم سيلاب ورودى به آبخوان Table 6. Correlation between Monthly SDI Index of Qanats around the Mehriz Flood spreading with SPI Index and Volume of Flood into the Aquifer

\begin{tabular}{|c|c|c|c|c|c|c|c|c|}
\hline & & حجم سيلاب & حجم سيل با بإ & حجم سيل با 9 & شاخص SPI & تاخير با باه SPI & تاخير SPI & ماه تاخير SPI \\
\hline شاخص SDI & $\mathrm{r}$ & $-\cdot$ - TAY & - IQTH &.$/ 1 \pi$ &.$- / f^{a}$ &.$/ I T Y$ & $\cdot / \Gamma)$. & $.1 .+9$ \\
\hline صادق آباد & $P$ value &.$/ \mu q V$ & $\cdot / \cdot v e$ & $\cdot / N T V$ &.$/ . \Delta$. & .1 .99 &...$t$ &.$/ D 1 V$ \\
\hline شاخص SDI محمد & $\mathrm{r}$ & $-\cdot / \cdot \Lambda \cdot$ & $-\cdot /\left.I V\right|^{c}$ &.$- /$ KFV &.$- / . .4$ & $\cdot / T \Lambda S^{* 3 *}$ & .1 .99 & $\cdot / K F \cdot{ }^{*}$ \\
\hline آباد & $P$ value & $\cdot / A \cdot \Delta$ & - $/ \Delta 9$. & . lo &.$/ 94 r$ &.$/$. & . $/ \mu v$. &.$/ . .1$ \\
\hline شاخص SDI بغداد & $\mathrm{r}$ & $-\cdot / / \wedge 9$ & 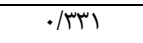 & $-. / 1+9$ & $-\cdot / K Y q^{* *}$ & $-\cdot / 19 \varphi^{*}$ &.$/ . \mu \cdot$ & $-\cdot / \cdot r q$ \\
\hline آباد & $P$ value & א אצץ/. & תוצ/. &.$|9 \Delta|$ &.$/ . r t r y$ &.$/ \cdot \Delta$ & . / $9 \wedge \mathrm{V}$ & $\cdot / 9 \cdot 9$ \\
\hline شاخص SDI & $\mathrm{r}$ &.$/ 1 F^{2}$ &.$/$. 99 & $-. / 4 \mid f$ & $-. / 1 / T$ &.$/ N{ }^{\prime}$ &.$- \cdot 1 \cdot .9$ & $-\cdot / \cdot F V$ \\
\hline حسين آباد & $P$ value & $.18 \Delta \Delta$ & (1/4 & $. / 1 \Lambda \mid$ &.$/ 1 r q$ & .1 .91 &.$/ 94$. & 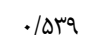 \\
\hline SDI شاخص & $\mathrm{r}$ & 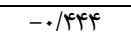 & $-\cdot / T \mu r$ & $-\cdot / 4 \cdot 9$ &.$- / 114$ & $-\cdot \cdot \cdot F \Delta$ & .1 .91 & .1 .90 \\
\hline ابراهيم آباد & $\mathrm{P}$ value &.$/ 1 Y A$ &.$/ 499$ &.$/ 191$ &.$/ M F F$ & . / QRq & . $/ \Gamma \Delta \Delta$ & $\cdot / r \cdot 1$ \\
\hline شاخص SDI & $\mathrm{r}$ & $\cdot / T \mid 9$ & $-\cdot / \cdot \mathrm{FV}^{\mathrm{F}}$ & - &.$/ . \% q$ & 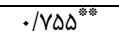 & $\cdot / \Gamma \wedge 9^{* 6 \%}$ &.$/ 199^{*}$ \\
\hline جشمه طزنج & $\mathrm{P}$ value & $\cdot 10 \cdot 1$ & - /MAD & . /TAY & س שqא/. &.$/ 099$ &.$/$ &.$/ . M F$ \\
\hline شاخص SDI & $\mathrm{r}$ & . &.$- / .9 Y$ &.$/ . \mu$. &.$/ .49$ & $-\cdot \cdot / \cdot \wedge r$ & $\cdot / \cdot r r$ &.$/ .4 q$ \\
\hline ارزاندازون & $\mathrm{P}$ value & $.10 \cdot 9$ & $\cdot / \mathrm{WV}$ & . /9TD &.$/ 090$ & . &.$/ 9 V T$ & $\cdot / V \cdot \Delta$ \\
\hline
\end{tabular}

جدول V- همبستخى بين شاخص SDI ماهانه قنوات اطراف يخش سيلاب مهريز با يكديخر

Table 7. Correlation between Monthly SDI Index of Qanats around the Mehriz Flood spreading

\begin{tabular}{|c|c|c|c|c|c|c|c|c|}
\hline & & حجم سيلاب & حجم سيل باه تاخير & حجم سيل باه & شاخص ماهانه & 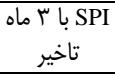 & $\begin{array}{c}\text { تاخير SPI } \\
\text { با }\end{array}$ & 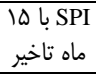 \\
\hline شاخص SDI & $\mathrm{r}$ & $-\cdot / T \wedge V$ & س TAH &.$/ 11 \%$ &.$- / 1 f^{4}$ & . MTr & $\cdot / r \mid \cdot{ }^{* 30}$ & $.1 .19 q$ \\
\hline صادق آباد & $P$ value & ./HGV & $\cdot 1 \cdot v^{c}$ & $\cdot / V T V$ &.$/ \cdot \Delta$. & .1 .98 & $\cdot 1+\varphi^{c}$ &.$/ D I V$ \\
\hline شاخص SDI محمد & $\mathrm{r}$ & $-\cdot / \cdot \Lambda \cdot$ & $-\cdot / I V^{4}$ & $-\cdot / T \& V$ & $-\cdot \cdot \cdot \cdot r$ & $\cdot / T \wedge \varphi^{* * 3 *}$ & .1 .99 & $\cdot /$ TF ${ }^{*}$ \\
\hline & $P$ value & $\cdot / \Lambda \cdot \Delta$ &.$/ 09$. & . &.$/ 914$ & .1. & $\cdot / r v \cdot$ & $.1+.1$ \\
\hline شاخص SDI بغداد & $\mathrm{r}$ & $-\cdot / / \wedge \wedge$ & ו וسץ/. & $-. / 1) 8$ & 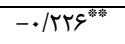 & $-\cdot / 19 \varphi^{\prime \prime \prime}$ & $\cdot / \cdot r \cdot$ & $-\cdot / \cdot r q$ \\
\hline آباد & $P$ value & אצץ/. & س & $\cdot|9 \Delta|$ & $.1 \cdot r$ & $\cdot / \cdot r \Delta$ & . I AAV & $\cdot 19 \cdot 9$ \\
\hline شاخص SDI & $\mathrm{r}$ &.$/ 1 F^{2}$ &.$/ 4 \& 9$ & $-\cdot / f \mid f$ &.$- / 114$ & $\cdot / 1 \mathrm{rV}$ &.$- \cdot 1 \cdot .9$ & $-\cdot / \cdot \mathrm{FV}^{\mathrm{V}}$ \\
\hline حسين آباد & $P$ value & $\cdot 19 \Delta \Delta$ & . / &.$/ 111$ &.$/ 1 r q$ & .1 .91 &.$/ 94$. & . /DFq \\
\hline شاخص SDI & $\mathrm{r}$ & $-0 . / 4 y^{2}$ & (-. & $-. \cdot / 4 \cdot 9$ &.$- / 11 \pi$ & $-. \cdot 1+4 \Delta$ & .1 .91 & .1 .90 \\
\hline ابراهيم آباد & $P$ value &.$/ 1 F A$ & $\cdot / 489$ &.$/ 191$ & . ITF & - / & $\cdot / r \Delta \Delta$ & $\cdot / \pi+1$ \\
\hline شاخص SDI & $\mathrm{r}$ & $\cdot / r \mid s$ & $-\cdot / \cdot c V$ & DT &.$/ .4 q$ & 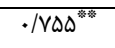 & $\cdot /{ }^{\prime} \wedge q^{3 *}$ &.$/ 199^{\prime \prime}$ \\
\hline جشمه طزنج & $\mathrm{P}$ value & $\cdot 10 \cdot 1$ & - /MND & . /TAV & . & .1098 &. & $.1 \cdot \mathrm{rF}$ \\
\hline شاخص SDI & $\mathrm{r}$ & (T/K & -.1 .94 &.$/ \cdot r$. & $\cdot / \cdot r q$ & $-\cdot / \cdot \wedge \mu$ & . & $.1 \cdot r q$ \\
\hline ارزاندازون & $P$ value & $.10 \cdot 9$ &.$/ \mathrm{V} V$ & ./9TQ & . $/ \Delta 9 \Delta$ & . &.$/ 9 V T$ & $\cdot / V \cdot \Delta$ \\
\hline
\end{tabular}


و همكاران (ه) نيز مطابقت دارد. اما نتايج نظر سنجى بهره

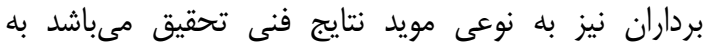

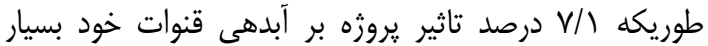

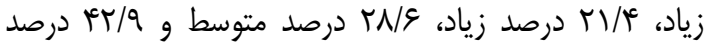

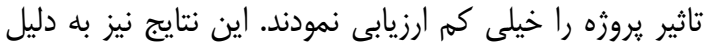

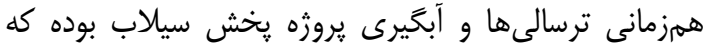

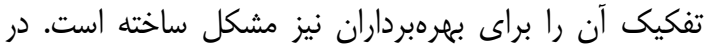

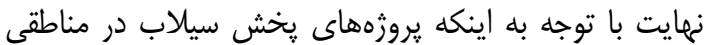

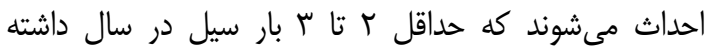

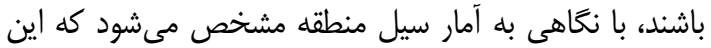

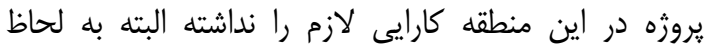

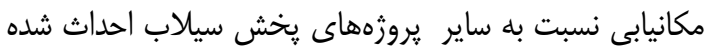

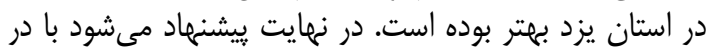

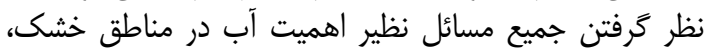

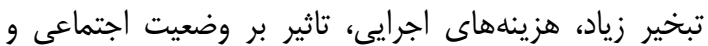

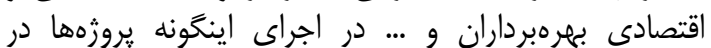

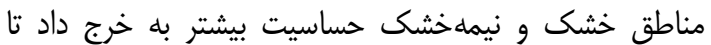
بتوان با بهترين روش بيشترين بازدهى را داشت.

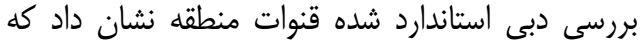

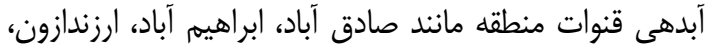

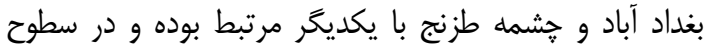

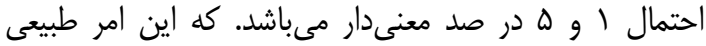

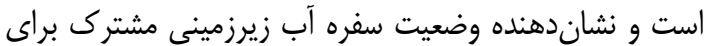

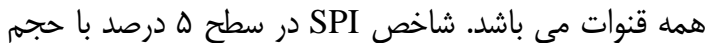

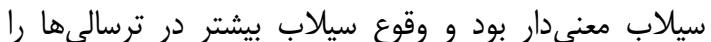

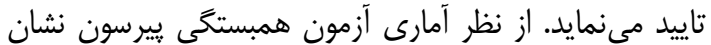

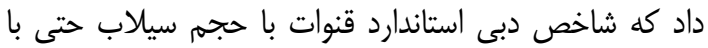

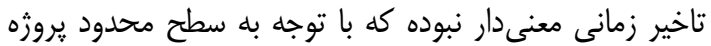

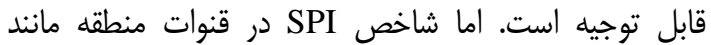

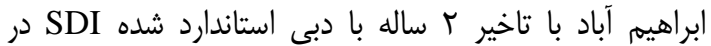

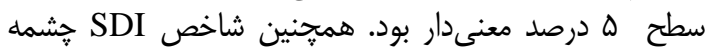

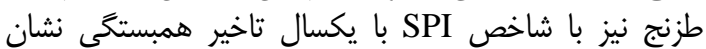

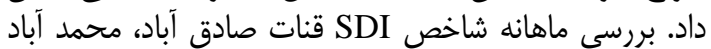

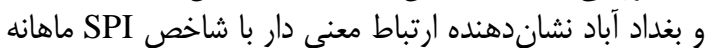

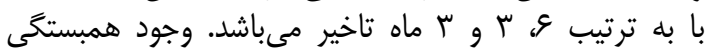

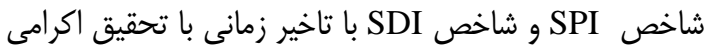

منابع Ghara Chryan Zanjan, Watershed Management Conference Proceedings, 96-90, (In Persian).

2. Bouri, S. and H. Ben Dhia. 2010. A Thirty- Year artificial recharge experiment in acoastal aquifer in an arid zone. The Teboulba aquifer system (Tunisian Sahel). CR Geosience, 342(1): 60-74.

3. Dadrasi, A. 1999. Climate and water resources limited in Sabzevar, Proceedings of the Second Regional Conference on Climate Change, IRIMO, 185-175. (In Persian).

4. Dadrasi Sabzevar, A. 2009. Assessment of reduce the effects of drought with floods, and artificial recharge projects. International Conference on Drought effects and management strategies (In Persian).

5. Ekrami, M., H. Maleki Nejad and M.r. Ekhtesasi. 2013. Study of the impact of climate and water droughts on groundwater resources (Case Study: Yazd-Ardakan plain), Iranian Journal of Watershed Management Science and Engineering, 4(20): 47-54 (In Persian).

6. Hashemi, S.A.A., B. Arasto and M. Ghodrati. 2011. Goosheh flood plain management's assessment of Damghan. The Sixth National Congress of Civil Engineering, University of Semnan, (In Persian).

7. Hashemi, H., R. Berendtsson and M. Persson. 2014. Artificial recharge by floodwater spreading estimated by water balances and groundwater modeling arid Iran, journal of hydrology science.

8. Katibe, H. and S. Hafezi. 2004. Modflow model using operational management and performance evaluation of artificial recharge of groundwater in Bam Abbarik plain. www.SID.ir.

9. Khalaji, M. 2000. Effects of flood spreading on the groundwater, the fourth conference of Geological Survey of Iran, (In Persian).

10. Kosar, A. 1995. Introduction to flood control and optimal use. Research Institute of Forests and Range.

11. Mckee, T.B., N.J. Doseken and J. Kleist. 1995. Drought monitoring with multiple time scales. Preprints, 9th conference, 15-20 January, Dallas, TX, 233-236.

12. Mirab, S.M., M. Hosseini, A. Atapoorfard and A. Khalilpour. 2005. Impact of Flood broadcasting stations on groundwater aquifers (Case Study: Aquifer station Shahyd hady Ahmadi in Tehran), Watershed Management and soil and water management Conference, Kerman, pp: 714-709 (In Persian).

13. Nahvinia, M., B. Karimi, H. Kardan moghadam and A. Shahidi. 2008. Technical and environmental evaluation of the effects of artificial flooding in Byrjand Syvan area. First International Conference on Water Crisis, (In Persian).

14. Sanjari, Gh.R. and Gh.h. Zorghi. 2001. Effect of flood spreading on the ground water level changes in Paskoh Saravan. Research and Construction of Natural Resources, 50: 54 to 57 (In Persian).

15. Shamshiri, Negar. 2003. The impact of flood spreading on the soil surface permeability changes (Case Study: Watershed Chandab Varamin), theses of the Master of Science, Tehran University (In Persian).

16. Sharifi, H. and M. Mahdian. 1999. Flood spreading is scientific solutions for efficient use of floods and feeding of aquifers, the first conference of the geology and the environment, (In Persian).

17. Soil Conservation and Watershed Management Research Center. 2011. Research and development strategy to take advantage of the flood of small water resources. Volume 1 to 4.

18. Viskarmy, A., P. Pyamny, A. Shah Karami and R. Sepahvand. 2013. The effect of flood spreading on Koohdasht groundwater resources. Journal of Science and Technology of Agriculture and Natural Resources, Soil and Water Sciences, 65: 163-160 (In Persian). 


\title{
The Effects of Flood Spreading on Qanat Discharge using Standard Discharge Index (Case Study: Qanats of Myankooh of Mehriz)
}

Mohammad Reza Fazel Pour aghdaei ${ }^{1}$, Hossein Malekinezhad ${ }^{2}$, Mohammad Reza Ekhtesasi $^{3}$ and Jalal Barkhordari ${ }^{4}$

\footnotetext{
1- PhD of Watershed Management, Department of Natural Resources and Watershed Management of Yazd, (Corresponding Author: fazelpoor_reza@yahoo.com)

2 and 3- Associate Professor and Professor, Department of Natural Resources and the desert of Yazd University 4- Faculty Member of Agriculture and Natural Resources Research Center of Yazd

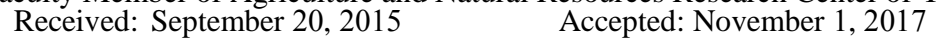

\begin{abstract}
Flood control, artificial recharge of aquifers and attempt to optimize the utilization of the natural resources are some of the most important measures in the arid and semi arid zones that occurs by flood spreading projects. At the current study the impact of flood spreading on downstream qanat discharge at Myankooh area of Mehriz (Yazd province) was investigated. To gain this goal after collecting the discharge Statistics from 1997 to 2013, Standard Discharge Index (SDI) was calculated. The amount of flood water entering the aquifer areas and precipitation figures were used to calculate the Standardized Precipitation Index (SPI). The correlation between the SDI and SPI and flood volume was extracted. Also, the operators' comments were collected by using the designed questionnaire. According to the results, the SDI increased at the same time as wet period and flood spreading time. But the share of each one on this invreasing wasn't clear. The correlation with SDI and SPI in different qanats was significant (1\%) with 12, 6 and 3 months' delay. Close to $28 \%$ operators assessed the project' impact high, $43 \%$ very low and others moderate.
\end{abstract}

Keywords: Aquifer, Recharge, Discharge, Flood, Yazd 